\title{
The clinical value of bedside ultrasound in predicting the severity of coronavirus disease-19 (COVID-19)
}

\author{
Jianzhong Xian ${ }^{1,2 \#}$, Xiaofeng Pei ${ }^{3 \#}$, Wuzhu Lu ${ }^{1}$, Haihong Zhong ${ }^{4}$, Yuhong Lin ${ }^{1}$, Hongjun Jin ${ }^{2}$, \\ Zhongzhen $\mathrm{Su}^{1,2}$
}

${ }^{1}$ Department of Ultrasound, Fifth Affiliated Hospital of Sun Yat-sen University, Zhuhai, China; ${ }^{2}$ Guangdong Provincial Key Laboratory of Biomedical Imaging and Guangdong Provincial Engineering Research Center of Molecular Imaging, Fifth Affiliated Hospital of Sun Yat-sen University, Zhuhai, China; ${ }^{3}$ Department of Thoracic Oncology, the Fifth Affiliated Hospital of Sun Yat-sen University, Zhuhai, China; ${ }^{4}$ Department of Radiology, Fifth Affiliated Hospital of Sun Yat-sen University, Zhuhai, China

Contributions: (I) Conception and design: Z Su, H Jin; (II) Administrative support: None; (III) Provision of study materials or patients: None; (IV) Collection and assembly of data: H Zhong; (V) Data analysis and interpretation: J Xian, X Pei, Y Lin; (VI) Manuscript writing: All authors; (VII) Final approval of manuscript: All authors.

\#These authors contributed equally to this work.

Correspondence to: Hongjun Jin; Zhongzhen Su. Guangdong Provincial Key Laboratory of Biomedical Imaging and Guangdong Provincial Engineering Research Center of Molecular Imaging, Fifth Affiliated Hospital of Sun Yat-sen University, Zhuhai, China. Email: jinhj3@mail.sysu.edu.cn; sp9313@126.com.

Background: To summarise the ultrasound manifestations of coronavirus disease-19 (COVID-19) patients with lung lesions and explore the clinical value of bedside ultrasound in the identification of patients at risk of progression to severe disease.

Methods: This retrospective study enrolled 31 patients with COVID-19 who were admitted to our hospital from January 18 to February 5, 2020. Lung ultrasounds were performed in all cases to evaluate the ultrasound manifestations of the patient's lung lesions and to determine the lung ultrasound scores (LUS). The Cox proportional hazards regression model was used for the multifactor analysis of 7 candidate parameters, including the LUS and the oxygenation index $\left(\mathrm{PaO}_{2} / \mathrm{FiO}_{2}\right)$. Receiver operating characteristic (ROC) curve analysis was performed to evaluate the predictive value of the LUS.

Results: Lung ultrasound images of COVID-19 patients mainly reflected the presence of interstitial pulmonary lesions $(90.3 \%, 28 / 31)$. The lung lesions were primarily distributed in the subpleural and peripheral pulmonary zones. Multivariate analyses identified the oxygenation index, the LUS, and the lymphocyte count as factors related to the progression to severe-critical disease in COVID-19 patients $(\mathrm{P}<0.05)$. With a cut-off value of 9.5 , the area under the ROC curve was 0.910 . The LUS showed a sensitivity and specificity of $81.3 \%$ and $93.0 \%$, respectively $(\mathrm{P} \leq 0.001)$, with an overall accuracy of $75 \%$.

Conclusions: The lung ultrasound findings in COVID-19 patients were mainly and specifically manifested as interstitial lesions involving the peripheral zones of the lung. In addition, ultrasound imaging could predict the likelihood of COVID-19 patients progressing to severe disease, thereby allowing for early intervention. Thus, lung ultrasounds have great clinical value in monitoring and evaluating COVID-19 patients.

Keywords: Coronavirus disease-19 (COVID-19); pneumonia; bedside ultrasound; lung ultrasound score (LUS); prediction; severe

Submitted Nov 29, 2020. Accepted for publication Jan 06, 2021.

doi: 10.21037/atm-20-7944

View this article at: http://dx.doi.org/10.21037/atm-20-7944

^ ORCID: Jianzhong Xian, 0000-0001-6643-9890; Hongjun Jin, 0000-0002-1522-1098. 


\section{Introduction}

Coronavirus disease-19 (COVID-19) is still a pandemic all over the world $(1,2)$. In some patients, the infection quickly progresses to severe or critical disease by one week after onset. Patients with severe disease present with dyspnoea and/or hypoxaemia, while the critically ill cases progress to acute respiratory distress syndrome, septic shock, refractory metabolic acidosis, coagulopathy, and multiple organ failure, which are difficult to treat and result in high mortality (3$5)$. Therefore, the timely identification of patients with critical disease is imperative for early intervention and is of great clinical importance for reducing the rate of mortality in coronavirus disease-19 (COVID-19) patients. However, a clinically effective method for identifying patients who show a high probability of progressing to severe disease is currently not available. At present, the main methods for identifying severe disease are examinations of blood oxygen saturation levels and chest computed tomography (CT) scans. These methods have obvious deficiencies, including the fact that lung lesions generally manifest before the appearance of low blood oxygen saturation levels (6). CT scans expose the patient to radiation and cannot be performed at the bedside. In addition, the conditions of patients can vary and deteriorate rapidly. Therefore, CT scans are not useful for timely and dynamic evaluations, especially for pregnant women and critically ill patients.

Transthoracic lung ultrasound (US) has recently become widely used due to its convenience, speed, safety, and ability to be applied for dynamic observations at the bedside (7-9). In the report of Lu et al. (10), the clinical significance for assessment and dynamic observation of lung lesions in patients with COVID-19 of bedside US was compared with CT, which showed that US has the similar performance in severe patients. This study aimed to explore the clinical value of bedside ultrasound for assessing the predisposition of patients in the early stages of the disease progressing to severe COVID-19. This will enable timely interventions to be administered. We present the following article in accordance with the STARD reporting checklist (available at http://dx.doi.org/10.21037/atm-20-7944).

\section{Methods}

\section{Institutional board approval}

All procedures performed in this study involving human participants were in accordance with the Declaration of Helsinki (as revised in 2013). This retrospective study was approved by our institutional review board and did not involve risks to the patients (Ethical code: 2020, L033-1). Patient written informed consent was waived.

\section{Patients}

Patients with COVID-19 admitted to our hospital from January 18 to February 5, 2020, were selected as research participants. The inclusion criteria were as follows: (I) positive results on SARS-CoV-2 nucleic acid assays (realtime reverse-transcriptase-polymerase-chain-reaction); (II) imaging examinations suggestive of pneumonia; and (III) no other viral pneumonia. The exclusion criteria were as follows: (I) positive history of chronic obstructive pulmonary disease or interstitial lung disease; (II) unable to undergo ultrasound examination; and (III) poor-quality bedside ultrasound images that could not be used for image analysis.

Based on the Diagnosis and Treatment Plan of COVID-19 issued by the National Health Commission (7th ed., Chinese language) (3), the types of COVID-19 patients were defined as follows: (I) mild: mild clinical symptoms without signs of pneumonia on imaging; (II) common: fever, respiratory tract symptoms and other signs/symptoms, signs of pneumonia on CT imaging; (III) severe: respiratory distress with respiratory rates of 30 breaths/minute or more, resting oxygen saturation of $93 \%$ or less, oxygenation index $\left(\mathrm{PaO}_{2} / \mathrm{FiO}_{2}\right)$ of $300 \mathrm{mmHg}$ or less; and (IV) critical: respiratory failure requiring mechanical ventilation, shock, and other organ failure requiring intensive care unit monitoring and treatment.

\section{Research methods}

\section{Ultrasonography}

Ultrasonography was performed using a Philips Affinity 50 Colour Doppler Diagnostic Ultrasound System (Bothell, WA, USA) equipped with a C6-2 convex-array probe (bandwidth: 2-5 MHz) and an L12-5 linear-array probe (bandwidth: 5-12 MHz). The same sonographer, a physician with more than 10 years of clinical experience, performed all the bedside ultrasound examinations. The sonographer wore biosafety level 3 protective clothing for each examination. In strict compliance with the highest level of personal protective equipment, the sonographer wore a disposable cap, full-face piece protective respirator or a higher-level positive-pressure and face-mounted protective respirator, surgical mask (N95), protective suit, 2 pairs of disposable latex gloves, and disposable shoe 
Table 1 The possible related factors associated with the progression to severe-critical COVID-19

\begin{tabular}{lc}
\hline Factor & Number (unit) \\
Age & Number (year) \\
Gender & Female 0, male 1 \\
Smoking history & No 0, yes 1 \\
Lung ultrasound score (LUS) & Number \\
Oxygenation index & Number (mmHg) \\
Lymphocyte count & Number (G/L) \\
C-reactive protein & Number (mg/L) \\
Progression to severe-critical disease & Yes 0, no 1 \\
\hline
\end{tabular}

For smoking history, "No" indicates non-smokers, "Yes" indicates current smokers or ex-smokers.

covers. For every examination, the array probe was covered with disposable sterile covers and thoroughly cleaned and disinfected after the examination. The sonographer who conducted the examination changed his outer gloves after every examination. Following this infection-control protocol, the sonographer remained uninfected for the duration of the entire study.

Patients were placed in the supine, lateral decubitus, and prone positions for the examinations. For critically ill patients, scanning of the dorsal thorax was conducted in the prone position during sputum excretion.

Each hemithorax was divided into 6 regions. Anterior and posterior axillary lines were used as landmarks to divide each hemithorax into anterior, lateral, and posterior regions; and an axial line was used to divide the hemithorax into upper and lower regions. The 6 regions of each hemithorax (anterior superior, anterior inferior, lateral superior, lateral inferior, posterior superior, and posterior inferior) were denoted as R1-R6 and L1-L6, respectively.

The intercostal spaces were first scanned by a convex array probe with the probe kept perpendicular to the ribs in the longitudinal plane. The probe was then rotated 90 degrees for transverse scanning for a comprehensive examination of the lungs and deep lesions. Subsequently, the linear array probe was used for complementary scanning, focusing on the outer margins of the lungs and the suspected subpleural lesions that had been detected by the convex array probe. Static and dynamic images were saved, and the duration time of the examination was recorded. After the initial scan, each patient underwent 2-5 additional dynamic ultrasound examinations at intervals of
1-6 days (mean interval 3.5 days).

\section{Chest CT}

A uCT 760 (United Imaging, China), uMI 780 (United Imaging, China), and Precision 32 CT scanner (Campo Imaging, China) were used to performed chest CT. All patients were placed in supine position and hold their breath after inhaling when scanned. The scanned area ranged from the thoracic inlet to the lung base. The scanning parameters were: tube voltage $120 \mathrm{kV}$; automatic tube current modulation; algorithms for high-resolution image reconstruction.

\section{Laboratory examinations}

Data on the patient's oxygenation index $\left(\mathrm{PaO}_{2} / \mathrm{FiO}_{2}\right)$, lymphocyte counts, and C-reactive protein (CRP) levels were collected for analysis.

\section{Image analysis}

The acquired images were transmitted to a back-office workstation. An independent blind comparison was performed during the analysis of the ultrasound images by 2 experienced deputy chief radiologists. The imaging manifestations and image characteristics were recorded, and the radiologists determined lung ultrasound scores (LUS) using the following method as the study report described previously (11): 0 points (pleural line and A-line, <3 B-lines); 1 point ( $\geq 3$ B-lines); 2 points (coalescent B-lines); and 3 points (signs of pulmonary consolidation). The LUS was calculated as the sum of all the regional scores, with a range of $0-36$ points.

The candidate factors and their corresponding values that might be associated with progression to severe or critical COVID-19 are shown in Table 1. If the LUS prediction results were not consistent with the clinical classification, CT images were used as the gold standard to analyze the possible causes of LUS prediction failure.

\section{Statistical analysis}

SPSS Statistics for Windows, Version 25.0 (IBM, New York, USA) was used for statistical analyses. Continuous data conforming to normal distribution were expressed as means \pm standard deviation. The Cox regression analysis was used to assess the candidate factors with relation to disease progression. Receiver operating characteristic (ROC) curve analysis was used to determine the diagnostic performance of the predictors that had been identified 


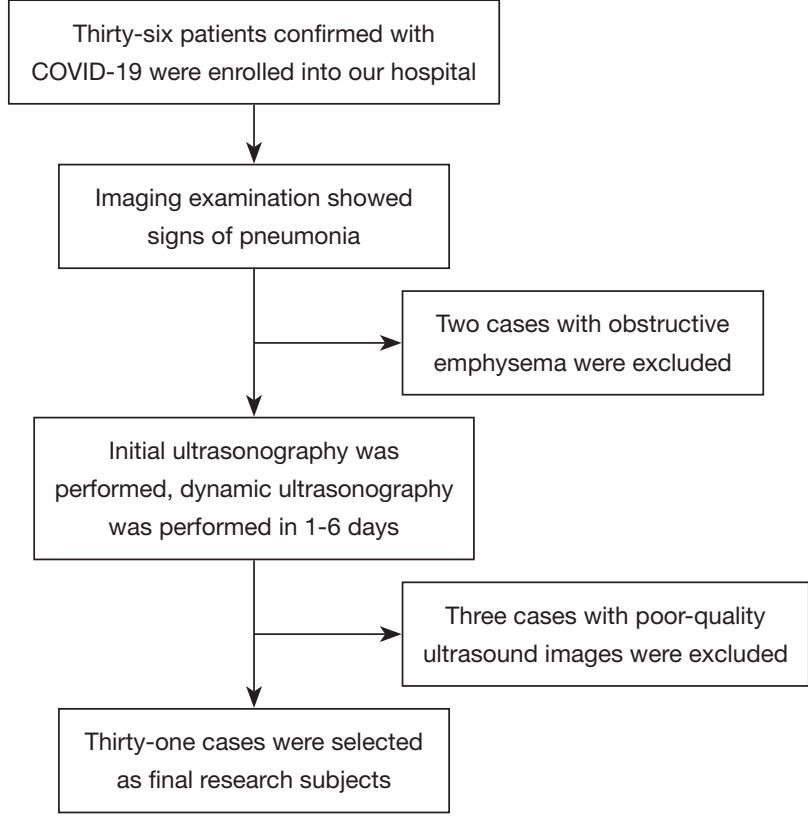

Figure 1 Enrolment procedure.

Table 2 Clinical characteristics of the patients

\begin{tabular}{lc}
\hline Characteristics & $\begin{array}{c}\text { Total number of patients } \\
(\mathrm{N}=31)\end{array}$ \\
\hline Gender & $17(54.8 \%)$ \\
Male & $14(45.2 \%)$ \\
Female & $56.5 \pm 14.9$ \\
Age (Year) & \\
Epidemiological history & $24(77.4 \%)$ \\
Recent travel to Hubei & $6(19.4 \%)$ \\
Exposure to infected people & $1(3.2 \%)$ \\
Unknown exposure & \\
Smoking history & $27(87.1 \%)$ \\
Non-smoker & $4(12.9 \%)$ \\
Current/former smoker & \\
Clinical signs/symptoms & $5(6.5 \%)$ \\
Normal & $3(9.7 \%)$ \\
Fever $\left(>37.5{ }^{\circ} \mathrm{C}\right)$ & $21(67.7 \%)$ \\
Cough & $15(48.4)$ \\
Weakness & $5(16.1 \%)$ \\
Nuscle soreness & \\
\hline & \\
Nausea and vomiting & \\
\hline
\end{tabular}

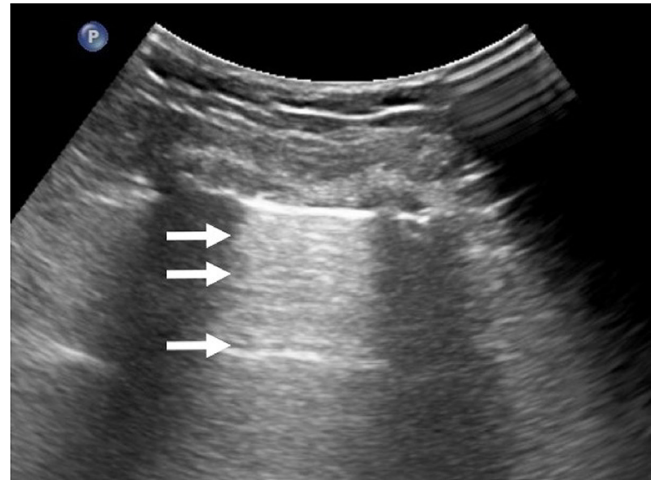

Figure 2 Lung ultrasound signs and lung ultrasound score (LUS) in a 50-year-old female. White arrows indicate A-lines and LUS $=0$.

as significant in the Cox analysis. The maximum value of the Youden index was determined and was used to select the cut-off value. Results with $\mathrm{P}<0.05$ were considered statistically significant.

\section{Results}

\section{Patients}

Figure 1 shows the inclusion process used to enroll patients in this study. A total of 31 patients with COVID-19 were included. None of the patients in the study cohort had mild COVID-19 disease. A total of 15 patients (48.4\%) were diagnosed with common type COVID-19, 11 (35.5\%) were diagnosed with severe disease, and 5 patients $(16.1 \%)$ had critical COVID-19. The clinical characteristics of all the study patients at the time of enrolment are shown in Table 2.

\section{Ultrasound findings}

During the study, the sonographer successfully completed bedside ultrasound examinations of all 31 patients. The duration of the examinations ranged from 5 to 8 minutes. Normal aeration on lung ultrasound and a LUS of zero were found in $3(9.6 \%$,) patients (Figure 2). A total of 28 patients $(90.3 \%)$ showed 3 B-lines or more $(90.3 \%)$ (Figure 3), of which 13 (41.9\%) patients showed coalescent B-lines (Figure 4). Pulmonary consolidation "debris sign" combined with "air bronchogram" were noted in 8 (25.8\%) patients (Figure 5). Thickening of the pleura was detected in $5(16.1 \%)$ patients, and small pleural fluid accumulations were found in $5(16.1 \%)$ patients. Pneumothorax was observed in $1(3.2 \%)$ patient. 
Bilateral and unilateral lung involvement was observed in 23 and 5 patients, respectively. A total of 144 lung regions showing signs of pneumonia were detected by ultrasound examinations. Most of the lesions detected by ultrasound were located in the R6, L6, L4, R4, and R3 regions, which indicated that the lesions were mainly distributed in the subpleural and peripheral zones of the lungs, with the lower lobe and dorsal region more likely to be involved.

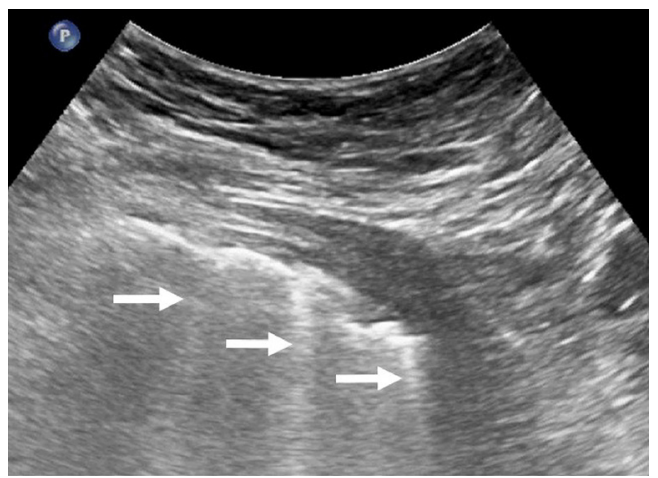

Figure 3 Lung ultrasound signs and lung ultrasound score (LUS) in a 75-year-old female. More than 3 B-lines were detected (white arrows) and LUS $=1$.

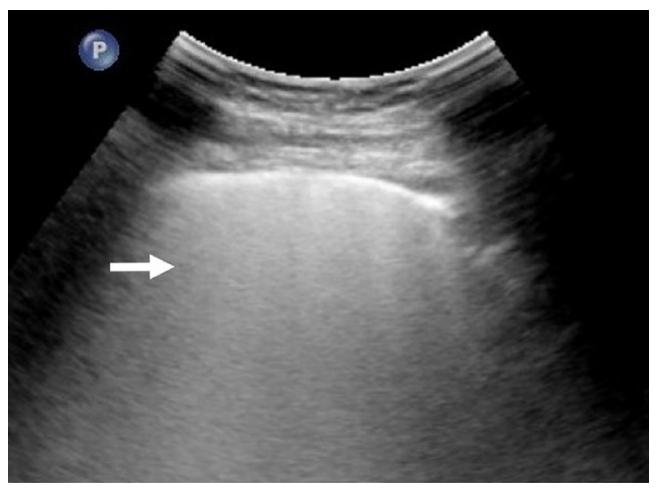

Figure 4 A lung ultrasound of a 38-year-old male, showing coalescent B-lines (white arrow) and a lung ultrasound score of 2 .

\section{Evaluation of the LUS as a predictor of progression to severe-critical COVID-19}

Seven predicative factors for progression to severe COVID-19 disease were included in the Cox proportional hazards regression analysis. The results suggested that the LUS, the oxygenation index, and the lymphocyte count were predictive factors of the progression of patients with COVID-19 to severe-critical disease. The significant $\chi^{2}$ values were $6.956,14.574,5.549$ respectively, as shown in Table 3.

The regression coefficient suggested that the higher the LUS, and the lower the oxygenation index and the lymphocyte count, the higher the probability that COVID-19 patients would progress to severe-critical disease.

The ROC curve analysis of the LUS is shown in Figure 6. With a cut-off score of 9.5 and an area under the ROC curve of 0.910, the LUS predicted that COVID-19 patients would progress to severe-critical disease with a sensitivity of $81.3 \%$ and a specificity of $93.0 \%(\mathrm{P} \leq 0.001)$.

With a LUS greater than 9.5 as the cut-off value, the accuracy of the LUS for predicting the progression of COVID-19 was analyzed. Data on 16 patients with

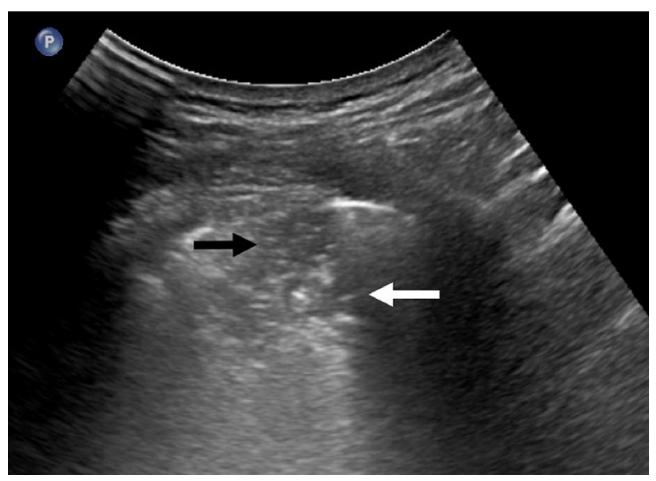

Figure 5 A lung ultrasound of a 64-year-old male, showing lung consolidations with "debris sign" (black arrow) and "air bronchogram" (white arrow), and a lung ultrasound score of 3.

Table 3 Significant predicative factors for the progression to severe COVID-19 disease as shown by Cox proportional hazards regression analyses

\begin{tabular}{|c|c|c|c|c|c|}
\hline Factors & $\mathrm{B}$ & Wald & $\mathrm{P}$ & $\mathrm{RR}$ & $\mathrm{RR} 95 \% \mathrm{Cl}$ \\
\hline LUS & 0.237 & 6.956 & 0.010 & 0.789 & $(0.658,0.945)$ \\
\hline Oxygenation index & -0.036 & 14.574 & $\leq 0.001$ & 0.965 & $(0.947,0.983)$ \\
\hline Lymphocyte count & -1.683 & 5.549 & 0.018 & 0.186 & $(0.046,0.754)$ \\
\hline
\end{tabular}

LUS, lung ultrasound score; $\mathrm{B}$, regression coefficient; Wald, $\chi^{2}$ value; RR, relative risk; $\mathrm{Cl}$, confidence interval. 


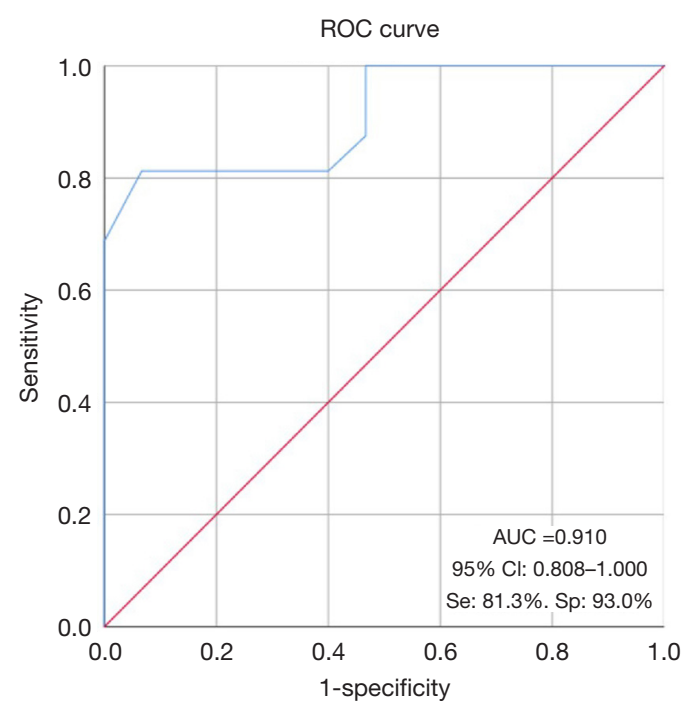

Figure 6 Receiver operating characteristic (ROC) curve of the lung ultrasound scores (LUS). The LUS predicted that COVID-19 patients would progress to severe-critical disease with a sensitivity (Se) of $81.3 \%$ and specificity $(\mathrm{Sp})$ of $93.0 \%$, and an area under the curve (AUC) of 0.910. CI, confidence interval. severe-critical COVID-19 are shown in Table 4. A total of 5 patients were classified with severe type disease upon admission, and 4 of these patients had LUS values greater than 9.5. Therefore, the accuracy of the LUS for predicting the progression of COVID-19 patients to severe type disease was $80.0 \%(4 / 5)$.

The 11 other severe-critical COVID-19 patients were classified with common type disease upon admission. All these patients progressed to severe-critical type disease within 3-9 days. Dynamic ultrasound examinations in 8 patients showed that the LUS value progressed to greater than 9.5, while the LUS value remained less than 9.5 in the other 3 patients. Therefore, the accuracy of the LUS for predicting the predisposition of COVID-19 patients progressing to severe disease was $72.7 \%$ (8/11). Among the 8 patients, the duration between the LUS value measuring greater than 9.5 to severe-critical disease was 1-3 days.

The overall accuracy of the LUS for predicting the progression of COVID-19 patients to severe type disease was $75.0 \%(12 / 16)$.

The LUS failed to predict the progression to severe

Table 4 The relationship between the LUS and patients progressing to severe-critical COVID-19

\begin{tabular}{|c|c|c|c|c|}
\hline $\begin{array}{l}\text { Severe-critical } \\
\text { COVID-19 patients }\end{array}$ & Clinical type at admission & Initial LUS & $\begin{array}{l}\text { Highest LUS value on } \\
\text { dynamic ultrasound }\end{array}$ & $\begin{array}{c}\text { Time from LUS reaching }>9.5 \\
\text { to progression to severe-critical } \\
\text { disease (days) }\end{array}$ \\
\hline 2 & Severe type & 6 & 17 & - \\
\hline 3 & Common type & 10 & 16 & 2 \\
\hline 5 & Common type & 3 & 18 & 2 \\
\hline 6 & Severe type & 18 & 18 & 0 \\
\hline 7 & Common type & 6 & 10 & 1 \\
\hline 8 & Common type & 0 & 12 & 3 \\
\hline 11 & Common type & 0 & 6 & - \\
\hline 12 & Common type & 18 & 25 & 2 \\
\hline 13 & Common type & 2 & 15 & 3 \\
\hline 14 & Severe type & 24 & 26 & 0 \\
\hline 15 & Common type & 0 & 6 & - \\
\hline 16 & Common type & 4 & 10 & 1 \\
\hline
\end{tabular}

-: indicates that the patient's LUS was $<9.5$ when they progressed to severe-critical type disease. LUS, lung ultrasound score. 

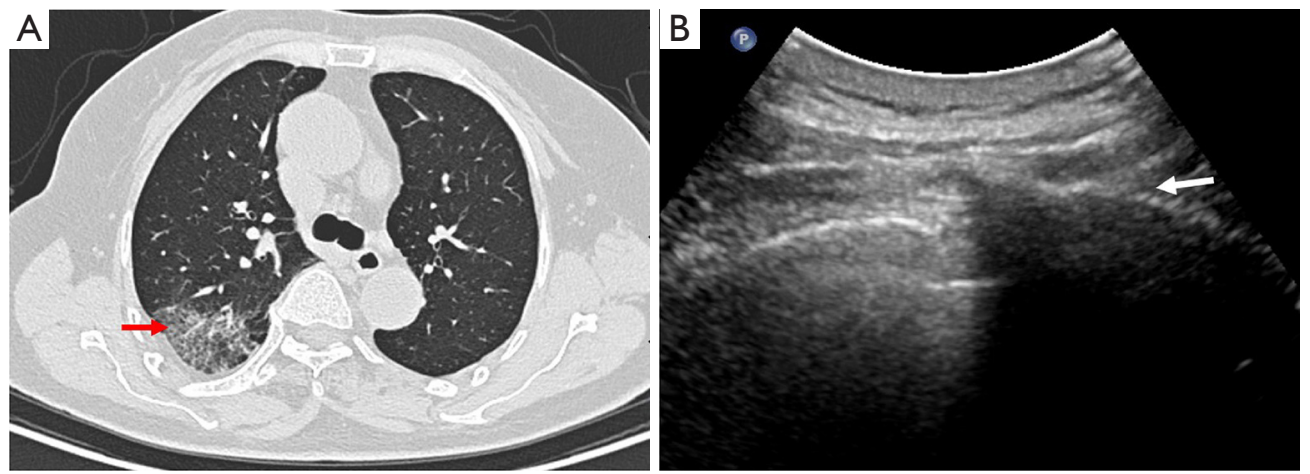

Figure 7 The lung ultrasound score (LUS) failed to predict progression to severe-critical disease in a 60-year-old female who presented with common type COVID-19 upon admission. She progressed to severe disease after 5 days, with a maximum LUS of 6 . (A) The lung computed tomography (CT) scan showed ground-glass opacities in the posterior segment of the right upper lobe (red arrow). (B) Ultrasound detection of that lung lesion was blocked by the scapula (white arrow). The ultrasound scan did not reveal the lesion.
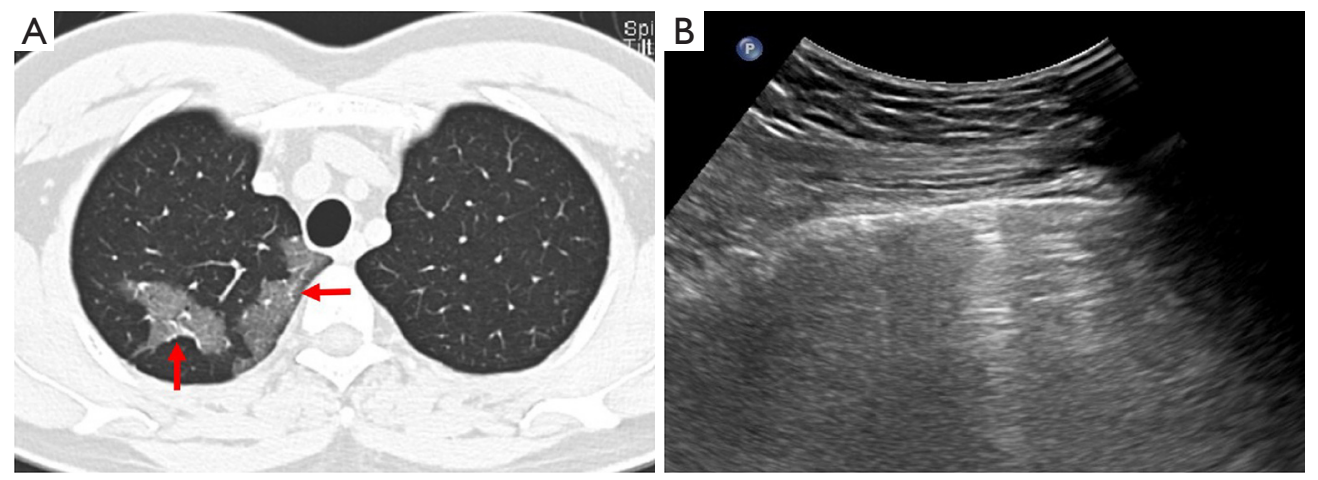

Figure 8 The lung ultrasound score (LUS) failed to predict progression to severe-critical disease in a 67-year-old male who was diagnosed with common type COVID-19 upon admission. He progressed to severe type disease after 4 days, with a maximum LUS of 7 . (A) The lung computed tomography (CT) scan showed ground-glass opacities (red arrows) in the tip of the upper lobe of the right lung. (B) The ultrasound scan did not reveal the lesion due to obstruction by lung tissue gases and the vertebra.

disease in 4 patients. A retrospective analysis found that the lung lesions of these patients were mainly located in the centre of the lung lobes, close the scapula or spine. They could not be visualised due to the surrounding gases of the normal lung tissue and the vertebrae. The ultrasound examinations failed to detect the lesions, thereby affected the LUS assessments (Figures 7-9).

\section{Discussion}

The new coronavirus pneumonia is an explosive infectious disease caused by the SARS-CoV-2 virus. The viral particles are very small, with diameters ranging from 60 to $140 \mathrm{~nm}$. When virus-containing droplets are inhaled, the virus can reach the alveolar septum, alveolar wall, lobular septum, and other terminal lung structures. Infection leads to extensive pulmonary interstitial oedema and lymphocytic infiltration. Early alveolar proteinaceous exudation in COVID-19 pneumonia is not obvious, but the disease progresses rapidly (12). Therefore, the early evaluation of lung lesions and the prediction of disease outcomes are important. As a non-invasive imaging evaluation method, transthoracic lung ultrasound has become widely used for clinical applications. It has been successfully applied to the evaluation of lungs affected by a variety of acute and chronic diseases (13).

The results of this study showed that the lung ultrasound examinations of COVID-19 patients revealed increased B-lines $(90.3 \%, 28 / 31)$ and coalescent B-lines $(41.9 \%$, 

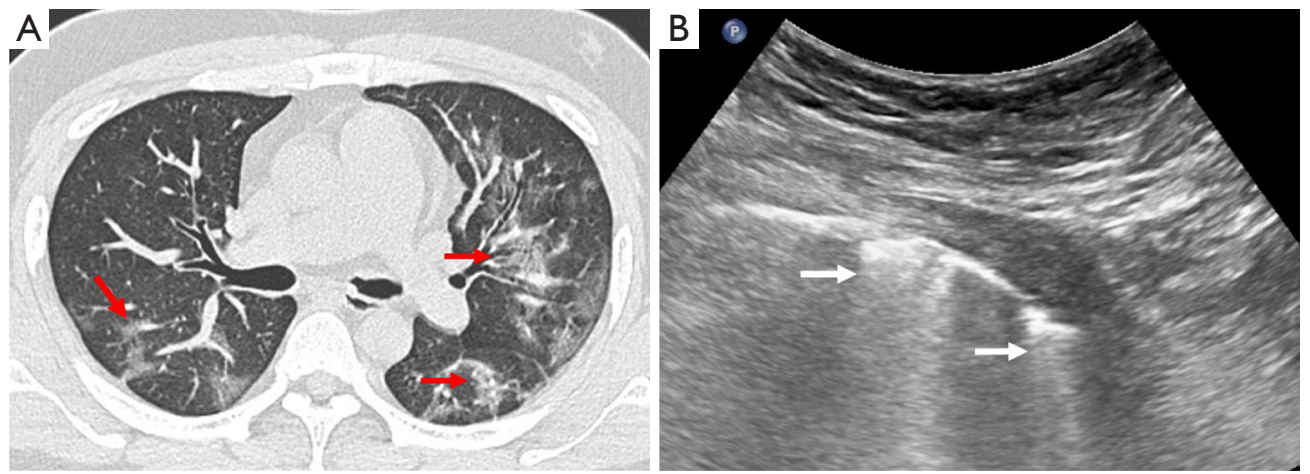

Figure 9 The lung ultrasound score (LUS) failed to predict progression to severe-critical disease in a 36-year-old male patient who presented with severe type COVID-19 upon admission. His initial LUS was 6 and the maximum LUS attained was 17. (A) The lung computed tomography (CT) scan showed that the majority of lesions were localized in the middle of lung (red arrows). (B) Only a small portion were localized in the peripheral zone. Ultrasonography showed more than 3 B-lines and coalescent B-lines (white arrows).

13/31). Most of the lesions were found on the lateral and dorsal sides of the lungs (R6, L6, L4, R4, and R3 regions), which were mainly distributed in the subpleural and peripheral zones of the lungs. These results were similar to those reported in previous CT studies (14-16). The B-lines are unspecific ultrasound artifacts generated by the physical interaction between the ultrasound beam and the different structures encountered by it from the chest wall to the lung air surface. The B-lines indicate the presence of lesions in the lung interstitium (17-19). An increased number of B-lines, coalescent B-lines, and signs of consolidation such as the "debris sign" and "air bronchogram" suggest an increased degree of lung lesions. The more extensive the lesions, the more severe the lung damage, which suggests that ultrasounds can evaluate lung damage in COVID-19 patients and provide technical evidence for the early prediction of the risk of severe COVID-19.

To clarify the clinical value of ultrasound for the early prediction of progression to severe-critical COVID-19, this study analyzed the relevant predictive factors for progression to severe-critical disease. The study identified 3 parameters, oxygenation index, LUS, and lymphocyte count, which were significantly related to the progression of COVID-19 patients to severe-critical disease $(\mathrm{P}<0.05)$.

The Wald statistic for the oxygenation index was the highest of the 3 factors (Wald statistic $=14.574$ ). The oxygenation index directly reflects lung function and has the closest relationship with the clinical condition of a patient, and as such, it has been used as a diagnostic indicator for COVID-19 classification (3). However, because of the strong compensatory ability of lung ventilation, the oxygenation index may remain normal or only decrease slightly, even though parts of the lung may be damaged by inflammation. The study by Caltabeloti et al. (6) showed that the oxygenation index decreased after changes in the ventilatory volume of the lung. The injury to the lung, due to COVID-19 pneumonia, was already very severe when low $\mathrm{PaO}_{2} / \mathrm{FiO}_{2}$ levels were detected, and the opportune moment to intervene with the best treatment was therefore often missed. Therefore, the oxygenation index should not be used to predict the predisposition of COVID-19 patients progressing to severe disease. In our study, (10) transthoracic lung ultrasounds were able to assess the loss of lung aeration, and the LUS provided a quantitative evaluation of total lung function. It showed the potential for quantifying the volume of lung ventilation and predicting disease progression. Ultrasound has the advantages of convenience, ease of performance, freedom from radiation exposure, and is capable of dynamic monitoring. The LUS showed a Wald statistic of 6.956, which was lower than the Wald statistic of $\mathrm{PaO}_{2} / \mathrm{FiO}_{2}$. However, it was able to identify patients who progressed to severe disease within 1-3 days of the initial or subsequent dynamic ultrasound examinations. Therefore, the LUS may allow for early clinical intervention. In addition, the LUS cut-off value of more than 9.5 for predicting the progression of COVID-19 patients to severe-critical disease had a sensitivity and specificity of $81.3 \%$ and $93.0 \%$, respectively, with an overall accuracy of $75 \%$. In the other hand, the LUS failed to predict the progression to severe disease in 4 patients, the reasons may be as follows. First of all, since the ultrasound could not visualize lesions located in the hilum, central 
lobes, subscapular region, or near the vertebral column due to obstruction by respiratory gases and bones, the LUS is much lower than the actual situation. So that the predictive performance of the LUS for these patients was adversely affected. Secondly, the dynamic ultrasound was not timely enough. For one patient in our study, the highest LUS was 17 , however it was not evaluated at the time when the patient become severe disease. So in order to improve predictive accuracy, we should perform the ultrasound examination very carefully and comprehensively.

This study has several limitations. First, the sample size was small and a larger sample size in a multicentre study is needed to support these results. Second, as mentioned above, ultrasound detection of lesions located in the centre of the lungs, especially near the hilum, is limited and this reduced the predictive accuracy of the LUS. Finally, this study was a retrospective analysis and future prospective studies are warranted.

In conclusion, bedside lung ultrasound demonstrated excellent clinical value for the monitoring and evaluation of COVID-19 patients. It was able to predict the likelihood of a COVID-19 patient progressing to severe disease, thus enabling clinicians to perform early interventions, which could reduce the mortality rate of COVID-19.

\section{Acknowledgments}

Funding: This work was financially supported by the National Science Foundation of China (No. 81871382), Fund for Emergency Study on Prevention and Control of COVID-19 Epidemic (ZH22036302200036PWC) and the Science and Technology Project of Zhuhai, China (No. 20181117E030025, 2018).

\section{Footnote}

Reporting Checklist: The authors have completed the STARD reporting checklist. Available at http://dx.doi. org/10.21037/atm-20-7944

Data Sharing Statement: Available at http://dx.doi. org/10.21037/atm-20-7944

Conflicts of Interest: All authors have completed the ICMJE uniform disclosure form (http://dx.doi.org/10.21037/atm-207944). The authors have no conflicts of interest to declare.

Ethical Statement: The authors are accountable for all aspects of the work in ensuring that questions related to the accuracy or integrity of any part of the work are appropriately investigated and resolved. All procedures performed in this study involving human participants were in accordance with the Declaration of Helsinki (as revised in 2013). This retrospective study was approved by our institutional review board and did not involve risks to the patients (Ethical code: 2020, L033-1). Patient written informed consent was waived.

Open Access Statement: This is an Open Access article distributed in accordance with the Creative Commons Attribution-NonCommercial-NoDerivs 4.0 International License (CC BY-NC-ND 4.0), which permits the noncommercial replication and distribution of the article with the strict proviso that no changes or edits are made and the original work is properly cited (including links to both the formal publication through the relevant DOI and the license). See: https://creativecommons.org/licenses/by-nc-nd/4.0/.

\section{References}

1. Cucinotta D, Vanelli M. WHO Declares COVID-19 a Pandemic. Acta Biomed 2020;91:157-60.

2. Spinelli A, Pellino G. COVID-19 pandemic: perspectives on an unfolding crisis. Br J Surg 2020;107:785-7.

3. Li K, Fang Y, Li W, et al. CT image visual quantitative evaluation and clinical classification of coronavirus disease (COVID-19). Eur Radiol 2020;30:4407-16.

4. Zhu N, Zhang D, Wang W, et al. A Novel Coronavirus from Patients with Pneumonia in China, 2019. N Engl J Med 2020;382:727-33.

5. Huang C, Wang Y, Li X, et al. Clinical features of patients infected with 2019 novel coronavirus in Wuhan, China. Lancet 2020;395:497-506.

6. Caltabeloti F, Monsel A, Arbelot C, et al. Early fluid loading in acute respiratory distress syndrome with septic shock deteriorates lung aeration without impairing arterial oxygenation: a lung ultrasound observational study. Crit Care 2014;18:R91.

7. Soldati G, Demi M, Smargiassi A, et al. The role of ultrasound lung artifacts in the diagnosis of respiratory diseases. Expert Rev Respir Med 2019;13:163-72.

8. Moro F, Buonsenso D, Moruzzi MC, et al. How to perform lung ultrasound in pregnant women with suspected COVID-19. Ultrasound Obstet Gynecol 2020;55:593-8.

9. Peng QY, Wang XT, Zhang LN. Findings of lung 
ultrasonography of novel corona virus pneumonia during the 2019-2020 epidemic. Intensive Care Med 2020;46:849-50.

10. Lu W, Zhang S, Chen B, et al. A Clinical Study of Noninvasive Assessment of Lung Lesions in Patients with Coronavirus Disease-19 (COVID-19) by Bedside Ultrasound. Ultraschall Med 2020;41:300-7.

11. Soummer A, Perbet S, Brisson H, et al. Ultrasound assessment of lung aeration loss during a successful weaning trial predicts postextubation distress* . Crit Care Med 2012;40:2064-72.

12. Kim EA, Lee KS, Primack SL, et al. Viral pneumonias in adults: radiologic and pathologic findings. Radiographics 2002;22 Spec No:S137-49.

13. Wallbridge P, Steinfort D, Tay TR, et al. Diagnostic chest ultrasound for acute respiratory failure. Respir Med 2018;141:26-36.

14. Chung M, Bernheim A, Mei X, et al. CT Imaging Features of 2019 Novel Coronavirus (2019-nCoV). Radiology 2020;295:202-7.

15. Bernheim A, Mei X, Huang M, et al. Chest CT Findings

Cite this article as: Xian J, Pei X, Lu W, Zhong H, Lin Y, Jin $\mathrm{H}, \mathrm{Su} Z$. The clinical value of bedside ultrasound in predicting the severity of coronavirus disease-19 (COVID-19). Ann Transl Med 2021;9(4):336. doi: 10.21037/atm-20-7944 in Coronavirus Disease-19 (COVID-19): Relationship to Duration of Infection. Radiology 2020;295:200463.

16. Zhou S, Wang Y, Zhu T, et al. CT Features of Coronavirus Disease 2019 (COVID-19) Pneumonia in 62 Patients in Wuhan, China. AJR Am J Roentgenol 2020;214:1287-94.

17. Hasan AA, Makhlouf HA. B-lines: Transthoracic chest ultrasound signs useful in assessment of interstitial lung diseases. Ann Thorac Med 2014;9:99-103.

18. Lomoro P, Verde F, Zerboni F, et al. COVID-19 pneumonia manifestations at the admission on chest ultrasound, radiographs, and CT: single-center study and comprehensive radiologic literature review. Eur J Radiol Open 2020;7:100231.

19. Yang W, Wang Y, Qiu Z, et al. Lung Ultrasound Is Accurate for the Diagnosis of High-Altitude Pulmonary Edema: A Prospective Study. Can Respir J 2018;2018:5804942.

(English Language Editor: J. Teoh) 